\title{
Atomic layering and related postmelting effects in small liquid metal clusters
}

\author{
Sara Núñez, José M. López, and Andrés Aguado* \\ Departamento de Física Teórica, Universidad de Valladolid, 47011 Valladolid, Spain \\ (Received 28 January 2009; revised manuscript received 31 March 2009; published 22 April 2009)
}

\begin{abstract}
The specific heat of $\mathrm{Na}_{25}$, as obtained by first-principles molecular dynamics simulations, shows interesting anomalies above the melting phase-change region: (a) It steadily decreases with increasing temperature, an effect observed also in bulk liquid metals. This trend is explained in terms of the gradual conversion of vibrational modes into diffusion modes; (b) on top of this decreasing trend, the specific heat shows broad undulations, induced by the temperature-dependent atomic layering in the liquid cluster. Extended liquid metal surfaces also show atomic layering, but it does not affect the bulk heat capacity. This effect is therefore genuine of finite atomic systems and should be expected to be quite general in metal clusters.
\end{abstract}

DOI: 10.1103/PhysRevB.79.165429

PACS number(s): 36.40.Ei, 31.15.xv, 61.25.Mv, 36.40.Mr

Melting of a macroscopic sodium sample is a first-order phase transition, which occurs at a well defined temperature for a given pressure. The corresponding meltinglike transition in sodium clusters is spread over a finite temperature interval and strongly depends on cluster size. Much effort has been invested in the past, both experimentally ${ }^{1-3}$ and theoretically, ${ }^{2-10}$ to understand the nature and size dependence of the solid-to-liquid transition and related premelting effects in sodium clusters. Much less attention has been paid, however, to the thermal properties of a cluster above its melting phase change region, where the liquidlike phase is fully developed. In this paper, we demonstrate that the heat capacity can show postmelting anomalies which reflect the underlying structural and dynamical properties of the liquidlike cluster. Some of them parallel the known bulk behavior, while some others are cluster specific.

The constant-volume heat capacity, $C_{v}$, of many bulk liquid metals decreases significantly with temperature: it has a value close to $3 N k_{B}$ at the melting point and steadily approaches $2 N k_{B}$ at higher temperature. ${ }^{11,12}$ The constantpressure heat capacity, $C_{p}$, shows a similar but smaller decrease upon heating. On the other hand, the liquid phase of metals is known to sustain high-frequency propagating collective modes (both longitudinal and transverse) down to wavelengths on the atomic scale, with solidlike dispersion relations. ${ }^{12}$ Computer simulations ${ }^{13-15}$ reveal substantial differences in the temperature evolution of longitudinal and transverse modes. The longitudinal modes are responsible for a high-frequency peak in the infrared vibrational spectrum. The transverse (shear) modes contribute to a low frequency peak which is continuously shifted toward zero frequency as temperature increases. This demonstrates that it is the gradual loss of shear resistance which induces the decrease in the liquid heat capacities. When the two transverse modes per atom are completely lost and only one longitudinal mode remains, we expect $C_{v}$ to be roughly equal to $2 N k_{B}$, if we exclude anharmonic corrections. The decrease in $C_{p}$ is less marked because the thermal expansion contribution competes with the loss of shear resistance. ${ }^{14,15}$ The gradual conversion of vibrational modes into diffusion modes upon heating has been captured in a phenomenological viscoelastic model by Trachenko. ${ }^{16}$ Specifically, those transverse modes with frequency $\omega<1 / \tau$, where $\tau$ is a liquid relaxation time, are converted into diffusion modes and therefore do not af- fect significantly the potential energy contribution to the heat capacity. As $\tau$ decreases with temperature, a larger proportion of transverse modes are lost. An alternative but physically equivalent interpretation has been given by Wallace, ${ }^{17}$ in terms of intervalley motions on the liquid potential energy surface (PES). When two anharmonic valleys intersect, there is a negative contribution to the heat capacity (called the boundary contribution by Wallace ${ }^{17}$ ) because moving along that direction does not increase anymore the potential energy. Due to the generality of this argument, the heat capacity decrease should be obeyed also by small liquid metal clusters.

There are also experimental ${ }^{18}$ and theoretical ${ }^{19-21}$ evidences for the existence of atomic layering at the surface of liquid metals. Chacón et al. ${ }^{19}$ have shown that the atomic layering is a general property of all materials with a low ratio between melting and critical temperatures. Alkali metals such as sodium satisfy this requirement. The low melting temperature provides stability for the cold liquid phases, with very stiff surfaces and small capillary wave (CW) amplitudes. For other (nonmetallic) liquids, the surface layering stage is usually not realized upon cooling because it is preempted by crystallization, although there can be exceptions. ${ }^{22}$ Metal clusters should be ideal systems to observe and study liquid surface layering for two reasons: (a) their melting points are usually depressed, so clusters offer access to colder liquid phases than bulk metals; (2) the CW thermal fluctuations produce a damping of the surface atomic structure, so that the mean density profile, sampled over a transverse area $A$, becomes more blurred for increasing $A .^{23}$ The very small size of a metal cluster removes most of the CW blurring effect ${ }^{24}$ and the layering can be more easily observed. The radial atomic density profiles of liquidlike $\mathrm{Na}_{139}$ and $\mathrm{Na}_{147}$ show indeed substantial layering. ${ }^{3}$ Here we show the same to be true even for very small clusters and analyze the possible impact of the atomic layering on thermal properties.

The simulations have been performed using orbital-free microcanonical molecular dynamics (OFMD), ${ }^{25}$ where density-functional theory is used to calculate the forces acting on the nuclei, whose motion is treated classically. An approximate density functional for the electron kinetic energy leads to a substantial simplification over the KohnSham (KS) method, enabling long simulations (up to 2-3 ns 


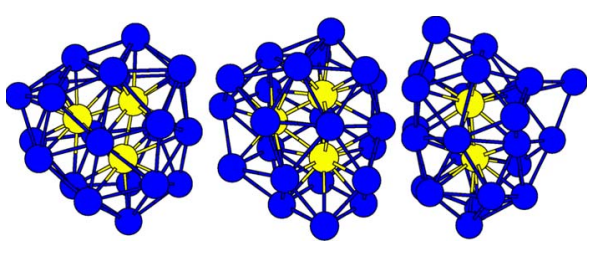

FIG. 1. (Color online) Left: putative global minimum structure of $\mathrm{Na}_{25}$; the two other plots show typical structures obtained by systematic quenching from the liquid phase, before (middle) and after (right) the postmelting anomaly is reached. The core atoms are shown in yellow (light gray) and the surface atoms are shown in blue (dark gray). Notice the change in the number of core atoms after the postmelting anomaly.

for each energy) to be performed. Such simulation lengths led to well converged potential energy distributions in the postmelting region which is of central interest to our work. All the computational details are the same as in our most recent works, ${ }^{9,10}$ where we also provide accuracy checks. The specific heat is obtained by the multiple histogram technique. ${ }^{26}$

The putative global minimum structure of $\mathrm{Na}_{25}$, shown in Fig. 1, has been obtained by simulated annealing, ${ }^{27}$ employing a cooling rate of $0.05 \mathrm{~K}$ per picosecond. The structure shows a polyicosahedral packing, with three interior atoms in a distorted icosahedral local environment, and the rest of atoms at the cluster surface. Our putative global minimum is not exactly the same but is very similar to the one obtained by Lee $e t ~ a l .{ }^{7}$ in KS calculations. We have additionally performed regular quenches (of the order of 200) starting from high-temperature OFMD trajectories, in order to have a sample of relevant local minima on the PES, and found that many structural isomers (including the global minimum of Lee et al.) exist within a narrow energy range of $2 \mathrm{meV} /$ atom. As we recently showed for $\mathrm{Na}_{30},{ }^{10}$ it is this global characterization of the PES which determines the gradual nature of melting in small sodium clusters, rather than the specific global minimum. The small differences between the $\mathrm{OF}$ and the KS structures is within the expected limits.

Microcanonical and canonical heat capacities are shown in Fig. 2. Both curves show a broad melting peak centered at about $T_{m}=150-160 \mathrm{~K}$, together with some premelting ( $T$ $\left.<T_{m}\right)$ and postmelting $\left(T>T_{m}\right)$ features. The microcanonical curve is more structured than the canonical one, ${ }^{28}$ a behavior explained by Doye ${ }^{29}$ in terms of the different functional dependences of microcanonical and canonical partition functions on their independent variables (energy and temperature, respectively). The canonical heat capacity can be compared with the KS results by Lee et al. ${ }^{7}$ The OF and KS heat capacities qualitatively agree in their shape, both showing a smooth and broad meltinglike transition, but the KS melting temperature is roughly $50 \mathrm{~K}$ above the OFMD result. The dynamical mechanisms leading to a gradual melting in small clusters have been analyzed in our previous work on $\mathrm{Na}_{30},{ }^{10}$ in terms of a continuous sequence of isomerizations involving the concerted motion of almost all atoms in the cluster. All those premelting details are very similar in $\mathrm{Na}_{25}$ and $\mathrm{Na}_{30}$, so they are not further discussed in this paper. Regarding the quantitative discrepancy, Calvo and co-workers ${ }^{30}$

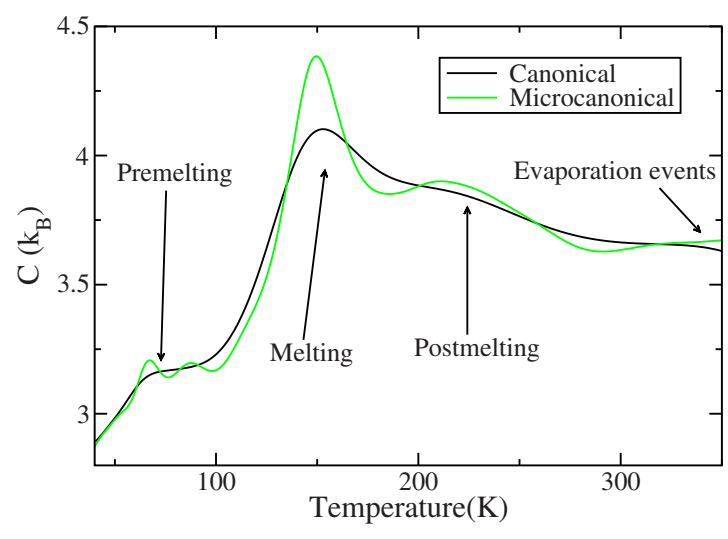

FIG. 2. (Color online) Canonical (black curve) and microcanonical (green or light gray curve) heat capacities per atom, shown in units of the Boltzmann constant. Notice the higher sensitivity of the microcanonical curve to structural changes.

have analyzed the dependence of the heat capacity curves on the number and length of the MD runs, providing explicit formulae which allow estimating the errors arising from incomplete sampling. Each OFMD simulation is more than 1 order of magnitude longer than the KS runs of Lee et al., and we have performed roughly three times more MD runs. Inserting this information into the expressions derived by Calvo, ${ }^{30}$ we estimate the statistical error of the KS simulations to be about $35 \mathrm{~K}$, which would explain most of the discrepancy. The remaining (small) difference is expected for different energy models.

We consider that the cluster is fully liquid when all atoms are able to diffuse and the cluster jumps between different local minima on the PES at a high rate. Structural indicators like the short-time-averages of the "atomic equivalence indexes," employed in our previous works, ${ }^{3}$ allow to visually identify how long the cluster remains on a given structural isomer. With the help of these indicators, we can safely state that the cluster is completely liquid at $20-30 \mathrm{~K}$ above $T_{m}$, that is, it is outside the melting phase change region. In this postmelting region, the heat capacity of the liquid cluster decreases on average, which is in good correspondence with the bulk trend and suggests that the explanation may be similar in both limits. However, different propagation directions are equivalent only in the bulk liquid metal due to its isotropy. For a cluster, it is not practical or even desirable to resolve the atomic velocities into components which are parallel and orthogonal to each possible propagation direction. But a natural and meaningful way of decomposing the atomic velocities in a cluster is into radial and tangential components. The radial component is defined for each atom as the projection of its velocity onto the direction which joins the atom position to the center of mass of the cluster; the tangential component is orthogonal to the same direction. Figure 3 shows the velocity autocorrelation functions (VACF) for the total, radial, and tangential velocities (averaged over all atoms in the cluster and normalized to their respective initial values) at two representative temperatures of the liquidlike cluster. At $T=180 \mathrm{~K}$, slightly above $T_{m}$, both radial and tangential VACFs show a damped oscillatory behavior, but the oscillations persist for much longer times in 


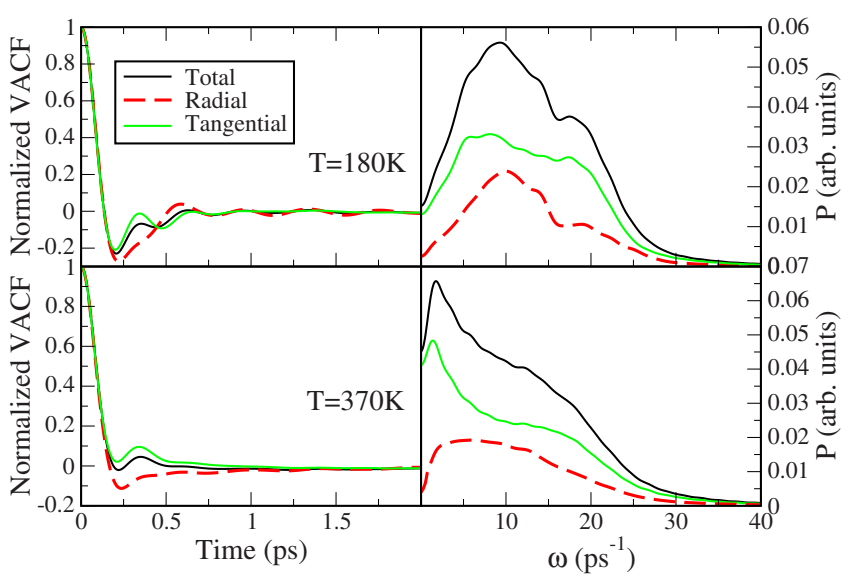

FIG. 3. (Color online) Velocity autocorrelation functions (left) and corresponding power spectra (right) for liquid $\mathrm{Na}_{25}$ at two representative temperatures, together with their partition into radial and tangential contributions. All VACFs are normalized to their respective initial values. The power spectra are not normalized, so the total spectrum is directly the sum of radial and tangential contributions. Note that there are two tangential and one radial mode per atom, hence the relative magnitudes of the corresponding power spectra.

the radial component. The radial velocity is therefore responsible for the long-time oscillatory tail of the total VACF. The first minimum, reflecting backscattering in atomic collisions, is deepest for the radial component, suggesting that radial diffusion is more impeded than tangential diffusion. The next maximum occurs at clearly different times in the radial and tangential components, contributing to two separate maxima in the total VACF and reflecting different dynamical behaviors along the radial and tangential directions. At $T=370 \mathrm{~K}$, the velocities lose memory of their initial values much faster, as expected. While the radial component continues to show backscattering, the tangential component never gets negative values, and approaches the simple exponential decay typical of weak confinement environments. The corresponding power spectra $P(\omega)$, obtained by Fourier transforming the VACFs, are shown on the right side of Fig. 3. $P(\omega)$ reflects the vibrational density of states of the liquid cluster, and its finite value at zero frequency reveals the existence of diffusion modes. As expected, the total spectral weight is shifted to lower frequencies with increasing temperature, but Fig. 3 shows that it is the tangential component which is mostly responsible for diffusion: upon heating, the radial power spectrum retains a very low value at zero frequency. Therefore, tangential modes are progressively lost upon heating, and this is reflected in the gradual decrease in the liquid heat capacity with temperature.

The liquid heat capacity shows anomalous undulations on top of the average decreasing trend, with a first broad postmelting peak centered at $210-220 \mathrm{~K}$ and a second one which is not fully developed because evaporation events ${ }^{31}$ set in at temperatures close to $380 \mathrm{~K}$. In order to understand the physical origin of these anomalies, we show in Fig. 4 the temperature evolution of the radial atomic distribution function $g(r)$, where $g(r) d r$ gives the average number of atoms at distances between $r$ and $r+d r$ from the center of mass of the

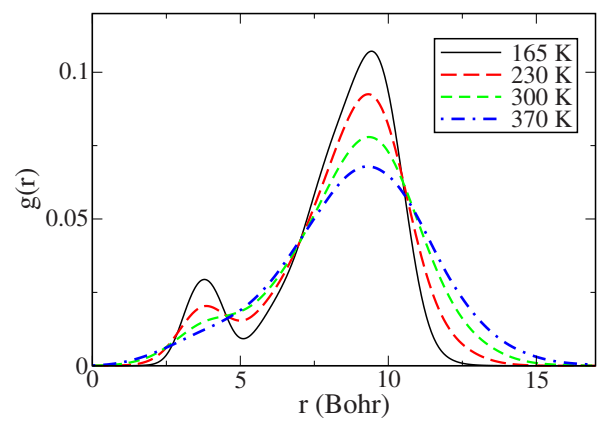

FIG. 4. (Color online) Radial atomic distribution function, $g(r)$, of liquid $\mathrm{Na}_{25}$ at four representative temperatures.

cluster. Close to the melting point, the atomic structure of the cold liquid shows surface layering, showing that the radial atomic packing is still far from random. Layering has also been recently observed in $\mathrm{Na}_{139}$ and $\mathrm{Na}_{147 .}{ }^{3}$ It is remarkable that extended surface properties persist in such small atomic systems. In the case of $\mathrm{Na}_{25}$, there are two radial atomic shells, which will be termed core and surface shells in the following. As seen in Fig. 4, the atomic layering gradually disappears upon heating, and at $T=370 \mathrm{~K} g(r)$ is almost a perfect parabolic function typical of random packing. We argue that it is the temperature evolution of the atomic layering profile which induces the postmelting anomalies in the liquid heat capacities. To check this idea, we have performed extensive and systematic quenching from runs performed below and above $220 \mathrm{~K}$, the temperature of the first postmelting peak. Below $220 \mathrm{~K}$, nearly all quenches result in isomers with three interior, corelike atoms; while above $220 \mathrm{~K}$, we mostly find isomers with only two corelike atoms (see Fig. 1 for typical examples). Each of these structural families of course consists of several isomers, spanning a finite energy interval, but the average energy of the less compact isomers is clearly higher, by approximately $10 \mathrm{meV} / \mathrm{atom}$. The bulk liquid heat capacity cannot depend on the atomic layering at the surface because the ratio of surface to bulk atoms is negligibly small; in a metal cluster, however, the promotion of one interior atom to the surface layer costs an amount of energy which is not negligible compared to the total binding energy and is therefore reflected as a broad peak in the cluster heat capacity. At higher temperatures, close to $370 \mathrm{~K}$, a similar analysis reveals that isomers with only one core atom are frequently visited, which is the reason for the emergence of the second undulation seen in Fig. 2.

In summary, we have shown that the average behavior of the liquid heat capacity, namely, a gradual decrease upon heating the liquid, is the same in a small metal cluster and in the bulk limit. A resolution of the liquid vibrational modes into radial and tangential components shows that it is the progressive conversion of tangential modes into diffusion modes which causes the heat capacity decrease. The liquid heat capacity of $\mathrm{Na}_{25}$ has a fine structure, with undulations on top of the average decreasing trend. These undulations are induced by the temperature dependence of the radial atomic layering, so they are cluster specific. The surface layering endows the cluster with a well-defined average radial structure, which in turn is characterized by a certain average en- 
ergy; so changes in that average radial structure are reflected in the heat capacity. Our calculations also reveal an interesting correlation between the temperature at which atomic layering disappears, the loss of long-time oscillations in the radial VACF, and a substantial increase in the evaporation rate. The presence of atomic layering is equivalent to an effective potential energy barrier acting against radial diffusion, which is naturally reflected in the backscattering oscillations of the radial VACF. For nonrotating clusters such as the one studied here, it is natural that evaporation becomes more prominent when radial diffusion is less impeded. It will be an interest- ing challenge for the experimentalists trying to resolve the fine structure in the liquid cluster heat capacities. The phenomena discussed in this paper should be quite general for any liquid cluster which shows atomic layering, as no sodium specific arguments have been employed, and emphasize the fundamental importance of atomic layering not only in extended liquids, but also in finite atomic clusters.

This research was supported by Junta de Castilla y León, the Spanish MEC, and the European Regional Development Fund (Projects No. FIS2008-02490/FIS and No. GR120). *aguado@metodos.fam.cie.uva.es

${ }^{1}$ H. Haberland, T. Hippler, J. Donges, O. Kostko, M. Schmidt, and B. von Issendorff, Phys. Rev. Lett. 94, 035701 (2005), and references therein.

${ }^{2}$ C. Hock, S. Straszburg, H. Haberland, B. v. Issendorff, A. Aguado, and M. Schmidt, Phys. Rev. Lett. 101, 023401 (2008).

${ }^{3}$ C. Hock, C. Bartels, S. Straszburg, M. Schmidt, H. Haberland, B. von Issendorff, and A. Aguado, Phys. Rev. Lett. 102, 043401 (2009).

${ }^{4}$ A. Rytkönen, H. Häkkinen, and M. Manninen, Phys. Rev. Lett. 80, 3940 (1998).

${ }^{5}$ F. Calvo and F. Spiegelman, Phys. Rev. Lett. 82, 2270 (1999); 89, 266401 (2002).

${ }^{6}$ S. M. Ghazi, M. S. Lee, and D. G. Kanhere, J. Chem. Phys. 128, 104701 (2008) and references therein.

${ }^{7}$ M. S. Lee, S. Chacko, and D. G. Kanhere, J. Chem. Phys. 123, 164310 (2005).

${ }^{8}$ A. Aguado, J. M. López, J. A. Alonso, and M. J. Stott, J. Chem. Phys. 111, 6026 (1999); J. Phys. Chem. B 105, 2386 (2001); A. Aguado, Phys. Rev. B 63, 115404 (2001); A. Aguado, L. M. Molina, J. M. López, and J. A. Alonso, Eur. Phys. J. D 15, 221 (2001).

${ }^{9}$ A. Aguado and J. M. López, Phys. Rev. Lett. 94, 233401 (2005); A. Aguado, J. Phys. Chem. B 109, 13043 (2005); E. G. Noya, J. P. K. Doye, D. J. Wales, and A. Aguado, Eur. Phys. J. D 43, 57 (2007).

${ }^{10}$ A. Aguado and J. M. López, Phys. Rev. B 74, 115403 (2006).

${ }^{11}$ R. N. Singh, S. Arafin, and A. K. George, Physica B 387, 344 (2007).

${ }^{12}$ W. C. Pilgrim and C. Morkel, J. Phys.: Condens. Matter 18, R585 (2006).

${ }^{13}$ T. Gaskell and S. Miller, J. Phys. C 11, 3749 (1978); 11, 4839 (1978).

${ }^{14}$ G. K. Straub, S. K. Schiferl, and D. C. Wallace, Phys. Rev. B 28, 312 (1983).

${ }^{15}$ M. Forsblom and G. Grimvall, Phys. Rev. B 72, 132204 (2005).

${ }^{16}$ K. Trachenko, Phys. Rev. B 78, 104201 (2008).

${ }^{17}$ D. C. Wallace, Phys. Rev. E 57, 1717 (1998).

${ }^{18}$ O. Shpyrko, P. Huber, A. Grigoriev, P. Pershan, B. Ocko, H. Tostmann, and M. Deutsch, Phys. Rev. B 67, 115405 (2003).

${ }^{19}$ E. Chacón, M. Reinaldo-Falagan, E. Velasco, and P. Tarazona, Phys. Rev. Lett. 87, 166101 (2001); E. Chacón, P. Tarazona, and L. E. González, Phys. Rev. B 74, 224201 (2006).
${ }^{20}$ D. J. González, L. E. González, and M. J. Stott, Phys. Rev. Lett. 92, 085501 (2004); 94, 077801 (2005).

${ }^{21}$ B. G. Walker, N. Marzari, and C. Molteni, J. Phys.: Condens. Matter 18, L269 (2006); J. Chem. Phys. 124, 174702 (2006); 127, 134703 (2007).

${ }^{22}$ H. Mo, G. Evmenenko, S. Kewalramani, K. Kim, S. N. Ehrlich, and P. Dutta, Phys. Rev. Lett. 96, 096107 (2006).

${ }^{23}$ A. Aguado, M. Wilson, and P. A. Madden, J. Chem. Phys. 115, 8603 (2001); A. Aguado, W. Scott, and P. A. Madden, ibid. 115, 8612 (2001); A. Aguado and P. A. Madden, ibid. 117, 7659 (2002).

${ }^{24}$ D. I. Zhukhovitskii, J. Chem. Phys. 125, 234701 (2006).

${ }^{25}$ A. Aguado, D. J. González, L. E. González, and J. M. López, in Trends in Chemical Physics Research, edited by A. N. Linke (Nova Science, New York, 2005), Chap. 10, p. 205.

${ }^{26}$ F. Calvo and P. Labastie, Chem. Phys. Lett. 247, 395 (1995).

${ }^{27} \mathrm{~A}$ total of ten independent simulated annealing runs was performed, three of which located the putative global minimum.

${ }^{28}$ The strong oscillations seen in the microcanonical heat capacity at about $70 \mathrm{~K}$ might be an artifact coming from broken ergodicity. In this region the cluster is fluctuating in time between several isomeric structures. The rate of isomer interconversion is sometimes sufficiently slow that the potential energy distributions may not be fully converged. The oscillations in the postmelting region are, however, reliable: here thermal equilibration is much faster and the potential energy distributions are well converged.

${ }^{29}$ J. P. K. Doye, Ph.D. dissertation, University of Cambridge, UK, 1996; J. P. K. Doye and D. J. Wales, J. Chem. Phys. 102, 9659 (1995).

${ }^{30}$ F. Calvo and C. Guet, J. Chem. Phys. 113, 1315 (2000); F. Calvo and D. J. Wales, ibid. 128, 154501 (2008).

${ }^{31} \mathrm{We}$ are not interested in a statistical description of evaporation processes in this paper. We simply stopped the simulations as soon as evaporation rates are high enough to be observed within our simulation time. This is the reason why we do not display the heat capacities for $T>350 \mathrm{~K}$. We note that evaporation events are observed at $380 \mathrm{~K}$, but the heat capacity is shown only up to $350 \mathrm{~K}$. The reason is that we only used those potential energy distributions which overlap significantly with other distributions obtained from runs at lower and higher energies, as required by the multihistogram analysis. 